PAPER

\title{
Medial temporal lobe atrophy in patients with refractory temporal lobe epilepsy
}

\author{
L Bonilha, E Kobayashi, C Rorden, F Cendes, L M Li
}

See Editorial Commentary, p 1606-7

J Neurol Neurosurg Psychiatry 2003;74:1627-1630

See end of article for authors' affiliations

.....................

Correspondence to: Dr L M Li, Department of Neurology, State

University of Campinas, UNICAMP, 13083-970, Campinas, SP, Brazil limin@fcm.unicamp.br

Received 4 April 2003 In revised form 4 June 2003 Accepted 9 June 2003
Objective: The objective of this study was to assess the volumes of medial temporal lobe structures using high resolution magnetic resonance images from patients with chronic refractory medial temporal lobe epilepsy (MTLE).

Methods: We studied 30 healthy subjects, and 25 patients with drug refractory MTLE and unilateral hippocampal atrophy (HA). We used T1 magnetic resonance images with $1 \mathrm{~mm}$ isotropic voxels, and applied a field non-homogeneity correction and a linear stereotaxic transformation into a standard space. The structures of interest are the entorhinal cortex, perirhinal cortex, parahippocampal cortex, temporopolar cortex, hippocampus, and amygdala. Structures were identified by visual examination of the coronal, sagittal, and axial planes. The threshold of statistical significance was set to $p<0.05$.

Results: Patients with right and left MTLE showed a reduction in volume of the entorhinal $(p<0.001)$ and perirhinal $(p<0.01)$ cortices ipsilateral to the HA, compared with normal controls. Patients with right MTLE exhibited a significant asymmetry of all studied structures; the right hemisphere structures had smaller volume than their left side counterparts. We did not observe linear correlations between the volumes of different structures of the medial temporal lobe in patients with MTLE.

Conclusion: Patients with refractory MTLE have damage in the temporal lobe that extends beyond the hippocampus, and affects the regions with close anatomical and functional connections to the hippocampus.
$\mathrm{T}$ he entorhinal cortex is considered to be the starting point of the basic circuitry involving the hippocampal formation because the information that reaches the hippocampus is tunnelled through it. ${ }^{1}$ The perirhinal, parahippocampal, and temporopolar cortices, in turn, provide the incoming connections to the entorhinal cortex, conveying information from polymodal and unimodal cortices. ${ }^{2}$

Damage to the medial temporal lobe region in patients with medial temporal lobe epilepsy (MTLE), as described by neuropathological studies, is not confined to the hippocampus, $^{3{ }^{4}}$ but extends to the parahippocampal region. Quantitative magnetic resonance imaging (MRI) studies have demonstrated that patients with MTLE show a significant reduction in the volume of the parahippocampal region ipsilateral to the side of hippocampal atrophy (HA). ${ }^{5-9}$ Nevertheless, it is not clear whether there is a uniform gradient of atrophy among patients with drug refractory MTLE, with regions closer to the hippocampus showing more degeneration. Moreover, there are conflicting data regarding which structures do present volume reduction in lateralised $\mathrm{TLE}^{6-10}$

The tight spatial arrangement of the medial temporal lobe structures poses a challenge for anatomical differentiation of cortical and subcortical structures based solely on gross surface landmarks. In this region of the brain, there is no straightforward correspondence between landmarks such as sulci or gyri and the boundaries defined by histology. ${ }^{11}$ This is particularly important because manual delineation of distinct regions with MR images relies mostly on anatomical landmarks. As each one of the relevant structures is generally composed of no more than a few cubic centimetres, minor imperfections in delineation may yield gross discrepancies and erroneous interpretation of important conclusions. With high resolution MRI imagery, this limitation can be minimised by using three dimensional visualisation ${ }^{12}$ of the relevant anatomical structures.
We assessed the degree of damage to the medial temporal lobe in consecutive patients with drug refractory MTLE through manual delineation of the cortical and subcortical structures of the medial portion of the temporal lobe in high resolution three dimensional MR images.

\section{METHODS}

Subjects

We studied 30 healthy adult subjects ( 19 women) without previous medical history of epilepsy. All subjects were contacted in the local community and were volunteers for this study. We also studied 25 consecutive patients with chronic refractory MTLE. All patients were referred from the outpatient epilepsy clinic of our institution, where they were diagnosed by a detailed neurological evaluation. The nature of the epileptic syndrome was determined based on ILAE criteria. ${ }^{13}$ Seizures were lateralised according to the medical history, a comprehensive neurological examination, interictal EEG, and prolonged video EEG monitoring of seizure onset. Visual inspection of the MRI scans following a standard protocol $^{14}$ revealed unilateral $\mathrm{HA}$ in all the patients. Each patient was diagnosed with drug refractory MTLE, ${ }^{15}$ with unilateral seizure onset. The ethics committee of our institution approved the study.

\section{MR image acquisition}

We acquired diagnostic MRI using a standardised protocol. ${ }^{14}$ $\mathrm{Tl}$ weighted images with $1 \mathrm{~mm}$ isotropic voxels were acquired on a 2 Tesla Elscint Prestige scanner (Haifa, Israel) using a spoiled gradient echo sequence ( $\mathrm{TR}=22 \mathrm{~ms}$,

Abbreviations: Amy, amygdala; Hip, hippocampus; ERC, entorhinal cortex; PHC, parahippocampal cortex; PRC, perirhinal cortex; ROI, regions of interest; TPC, temporopolar cortex 
$\mathrm{TE}=9 \mathrm{~ms}$, flip angle $=35^{\circ}$, matrix $=256 \times 220$, field of view $=25 \times 22 \mathrm{~cm}, 1 \mathrm{~mm}$ thick sagittal slices).

\section{Image post-processing}

We transferred the volumetric images to a Silicon Graphics O2 workstation (Mountain View, CA, USA). The raw images were initially converted into MINC format, corrected for field non-homogeneity using $\mathrm{N} 3,{ }^{16}$ and transformed into a standard space by linear stereotaxic transformation. ${ }^{17}$

\section{Volumetric analysis}

We performed manual segmentation using the interactive software package Display (David McDonald) developed at the Brain Imaging Centre of the Montreal Neurological Institute. This software enables precise and fully scalable image analysis by simultaneously displaying coronal, sagittal, and axial planes. It also makes it possible to draw regions of interest (ROI), and delineation of anatomical boundaries is facilitated by contrast adjustment and the possibility for navigation through isotropic voxels of $1 \mathrm{~mm}$ in different orientations with the same resolution. The software automatically calculates the volumes of the labelled structures.

The structures were manually delineated by a single observer (L B), according to previously published comprehensive reviews of the MRI anatomical boundaries of medial temporal lobe structures. ${ }^{6711} 1218$

We evaluated the volumes of the temporopolar cortex (TPC), perirhinal cortex (PRC), entorhinal cortex (ERC), parahippocampal cortex (PHC), amygdala (Amy), and hippocampus (Hip).

\section{Statistical analysis}

Data was evaluated using Systat (9.0) software for Windows. Group differences for age were evaluated using one way analysis of variance, and gender distribution was evaluated using the $\chi^{2}$ test. Differences of volumes among control subjects were evaluated with multivariate analysis of variance with two intra-subject factors (side (left or right) and structure (TPC, PRC, ERC, PHC, Amy, Hip)). The value of each individual's volume measurement was standardised according to the volumes of th mean observed in the controls group. For each individual, asymmetry of volumes was calculated according to the symmetrical index $(\mathrm{L}-\mathrm{R}) /$ $[(\mathrm{L}+\mathrm{R}) / 2]$. Group differences of volume were evaluated with multivariate analysis of variance with one inter-subject grouping factor (controls, left MTLE, right MTLE) and one intra-subject control group through Z score transformationthat is, the number of standard deviations (SD) away from that grouping factor (structures: right TPC, left TPC, right PRC, left PRC, right ERC, left ERC, right PHC, left PHC, right Amy, left Amy, right Hip, left Hip). The multivariate analysis of variance was followed by Tukey HSD post hoc comparisons to evaluate the structures that would exhibit significant volume reduction in patients with MTLE compared with normal controls.

The degree of atrophy across the different structures was performed using a one way analysis of variance to compare the $\mathrm{Z}$ scores of each medial temporal lobe structure. Group differences for asymmetry were evaluated with multivariate analysis of variance with one inter-subject grouping factor (controls, left MTLE, right MTLE) and one intra-subject grouping factor (TPC, PRC, ERC, PHC, Amy, Hip). We used correlation and simple regression to assess the interdependent variability of the volumes measured. The threshold for statistical significance was set at $p<0.05$. We considered the volume of the structure to be abnormally reduced when its $\mathrm{Z}$ score was below 2 SD.

\section{RESULTS}

Thirteen patients had left HA and left MTLE and twelve patients had right HA and right MTLE. There was no significant difference in age or sex distribution between controls and patients with MTLE.

\section{Control subjects}

The volumes of the different structures in of the control subjects are shown in table 1 . There was no significant side to side difference in any of the structures analysed $\left(\operatorname{PRC} \lambda_{(58,1)}=0.57, \mathrm{p}=0.45 ; \operatorname{TPC} \lambda_{(58,1)}=0.29, \mathrm{p}=0.59\right.$; ERC $\lambda_{(58,1)}=0.59, \mathrm{p}=0.44 ;$ PHC $\lambda_{(58,1)}=0.84, \mathrm{p}=0.36$; Amy $\lambda_{(58,1)}=1, \mathrm{p}=0.32$; Hip $\lambda_{(58,1)}=0.04, \mathrm{p}=0.84$.

\section{Patients with MTLE}

The volumes of the studied structures in patients with MTLE are shown in table 1. Multivariate analysis of variance with Tukey post hoc comparison between patients and controls showed a significant reduction in the volumes of the left PRC, left ERC, and left Hip in patients with left MTLE, and significant reduction in the volumes of the right PRC and right ERC in patients with right MTLE (fig l). Patients with left MTLE exhibited a larger right amygdala compared to controls and patients with right MTLE: $\lambda_{(52,2)}=4.6, p<0.05$.

\section{Asymmetry}

Group differences on asymmetry were significant for all structures analysed, as follows: PRC $\lambda_{(52,2)}=36.5, \mathrm{p}<0.001$ Tukey's: left MTLE $<$ control<right MTLE; TPC $\lambda_{(52,2)}=11.4$, $\mathrm{p}<0.001$, left $\mathrm{MTLE}=$ control $<$ right MTLE; ERC $\lambda_{(52,2)}=$ 106.9, $\mathrm{p}<0.001$, left $\mathrm{MTLE}<\mathrm{control}<$ right MTLE; PHC $\lambda_{(52,2)}=22.3, \mathrm{p}<0.001$, left $\mathrm{MTLE}=\mathrm{control}<$ right $\mathrm{MTLE}$; Amy $\lambda_{(52,2)}=8.5, \mathrm{p}<0.01$, left MTLE $=$ control $<$ right MTLE; Hip $\lambda_{(52,2)}=274.1, \quad \mathrm{p}<0.001$, left $\mathrm{MTLE}<$ control $<$ right MTLE.

\section{Individual analysis}

The analysis of the $\mathrm{Z}$ score revealed that the degree of atrophy was significantly different among the medial temporal lobe structures in patients with left MTLE $\mathrm{F}_{(13,11)}=26.45$, $\mathrm{p}<0.001$ and in patients with right MTLE $\mathrm{F}_{(12,11)}=22.22$, $\mathrm{p}<0.001$ (fig 2). Tukey post hoc comparison did not show difference between the $\mathrm{Z}$ scores of the ERC and the PRC in patients with left and patients with right MTLE.

\section{Correlation and simple regression}

Linear correlation and simple regression analysis were performed with the data from similar structures ipsilateral to HA-that is, left perirhinal cortex in patients with left MTLE and right perirhinal cortex in patients with right MTLE, grouped together. All structures were analysed simultaneously in the search for significant inter-structure correlation. There was no significant linear correlation between the volumes of structures analysed-that is, there was no inter-dependent variability between the volumes of the different structures of the medial temporal lobe.

Simple regression analysis revealed $R^{2}=0.3$ for the comparison between the entorhinal and perirhinal cortices. All other comparisons revealed smaller values of $\mathrm{R}^{2}$.

\section{DISCUSSION}

Volumetric analysis of the amygdala and hippocampus has been successfully used to determine tissue damage in patients with temporal lobe epilepsy. ${ }^{19}$ Currently, few studies have examined the other temporal lobe structures, particularly the cortical structures of the medial temporal lobe, as reviewed below. As there is a large and intricate network of connections involving all structures in the medial temporal lobe, it is possible that damage to the medial temporal lobe 
Table 1 Volumes of mesial temporal structures of normal subjects and patients with MTLE

\begin{tabular}{|c|c|c|c|c|c|c|c|c|c|c|c|c|c|}
\hline \multirow[b]{2}{*}{ Group } & \multirow[b]{2}{*}{ Structure } & \multicolumn{2}{|l|}{ PRC } & \multicolumn{2}{|l|}{ TPC } & \multicolumn{2}{|l|}{ ERC } & \multicolumn{2}{|l|}{ PHC } & \multicolumn{2}{|l|}{ Amy } & \multicolumn{2}{|l|}{ Hip } \\
\hline & & $\mathbf{L}$ & $\mathbf{R}$ & $\mathbf{L}$ & $\mathbf{R}$ & $\mathbf{L}$ & $\mathbf{R}$ & $\mathbf{L}$ & $\mathbf{R}$ & $\mathbf{L}$ & $\mathbf{R}$ & $\mathbf{L}$ & $\mathbf{R}$ \\
\hline \multirow[t]{4}{*}{ R MTLE } & Mean & 2140 & 1709 & 3337 & 2954 & 1159 & 856 & 1642 & 1331 & 1454 & 1335 & 3031 & 1764 \\
\hline & SD & 363 & 315 & 541 & 522 & 126 & 127 & 297 & 229 & 140 & 285 & 290 & 328 \\
\hline & Max & 2670 & 2247 & 4118 & 3924 & 1550 & 1066 & 2087 & 1786 & 1741 & 2065 & 3558 & 2305 \\
\hline & Min & 1527 & 1259 & 2492 & 1836 & 982 & 669 & 1201 & 1026 & 1239 & 987 & 2590 & 1137 \\
\hline \multirow{4}{*}{ L MTLE } & Mean & 1701 & 2083 & 2990 & 3314 & 802 & 1139 & 1522 & 1580 & 1479 & 1616 & 1823 & 3051 \\
\hline & SD & 290 & 350 & 524 & 584 & 119 & 153 & 259 & 206 & 240 & 178 & 293 & 312 \\
\hline & Max & 2182 & 2712 & 4032 & 4308 & 959 & 1363 & 2100 & 1857 & 1873 & 1871 & 2291 & 3520 \\
\hline & Min & 1225 & 1671 & 2189 & 2550 & 627 & 828 & 1185 & 1246 & 1090 & 1281 & 1385 & 2561 \\
\hline \multirow[t]{4}{*}{ Controls } & Mean & 2266 & 2187 & 3244 & 3173 & 1187 & 1226 & 1661 & 1573 & 1539 & 1479 & 3279 & 3260 \\
\hline & SD & 386 & 418 & 454 & 547 & 182 & 197 & 366 & 371 & 233 & 222 & 374 & 352 \\
\hline & Max & 3063 & 2922 & 4032 & 4282 & 1660 & 1897 & 2590 & 2572 & 1946 & 2175 & 3949 & 3999 \\
\hline & Min & 1315 & 1395 & 1987 & 1981 & 819 & 827 & 1227 & 1146 & 1046 & 1010 & 2458 & 2369 \\
\hline
\end{tabular}

R, right; L, left; PRC, perirhinal cortex; TPC, temporopolar cortex; ERC, entorhinal cortex; PHC, parahippocampal cortex; Hip, hippocampus; Amy, amygdala; Max, maximum; Min, minimum

may extend beyond the amygdala and the hippocampus, ${ }^{6}$ which may contribute to the symptoms of temporal lobe epilepsy.

It is not yet established to what extent damage to the medial temporal lobe involves the cortical structures. Jutila et $a l^{6}$ examined patients with unilateral MTLE and reported that patients with right HA have ipsilateral damage in the entorhinal and temporopolar cortices, whereas patients with left HA have ipsilateral damage only in the entorhinal cortex. Salmenpera et al ${ }^{10}$ observed that in patients with MTLE the mean volumes of the entorhinal cortex ipsilateral to the side of seizure onset did not differ from controls. However, they found that the entorhinal volume correlated with hippocampal volume and that patients with right MTLE with HA had a $19 \%$ volume reduction of the ipsilateral entorhinal cortex. In their study, the volume of the entorhinal cortex correlated with the duration of MTLE. They also found that none of the patients showed additional atrophy in the hippocampus, amygdala, or the entorhinal and perirhinal cortices at a 1 year follow up. ${ }^{18}$ However, Bernasconi et $a l^{8}$ have found volume reduction of the entorhinal cortex ipsilateral to the seizure focus in all MTLE patients studied, reduction of the perirhinal cortex in $33 \%$ of the patients, and contralateral reduction of the entorhinal cortex in $50 \%$ of the patients. The same group also found bilateral reduction of the entorhinal cortex volume in patients with MTLE, the atrophy being greater ipsilateral to the epileptic focus, ${ }^{7}$ and observed that it was possible to lateralise the seizure focus in patients with MTLE and normal hippocampal volumes based on the entorhinal cortex volume. ${ }^{9}$ Recently, they examined a group of 25 patients with drug refractory MTLE and observed that the entorhinal and the perirhinal cortices ipsilateral to the HA were significantly smaller than in normal controls. They also observed that the entorhinal cortex was more severely reduced than the perirhinal cortex. ${ }^{5}$

In summary, we analysed medial temporal lobe structures in consecutive patients with unilateral chronic refractory MTLE defined and documented by video EEG, along with unilateral HA. Our findings demonstrated that patients with MTLE have significant reduction of the volume of the cortical structures closer to the hippocampus - that is, the entorhinal and perirhinal cortices. Other structures, such as the

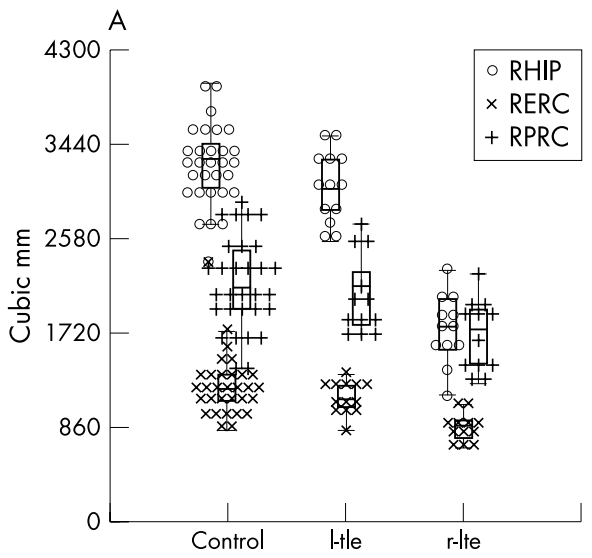

Right hippocampus

$\lambda=84.05, \mathrm{p}<0.001$.

Tukey's: RTLE < Control = LTLE

Right perirhinal cortex

$\lambda=6.55, p=0.01$

Tukey's: RTLE < Control = LTLE

Right entorhinal cortex

$\lambda=18.84, p<0.001$

Tukey's: RTLE < Control = LTLE

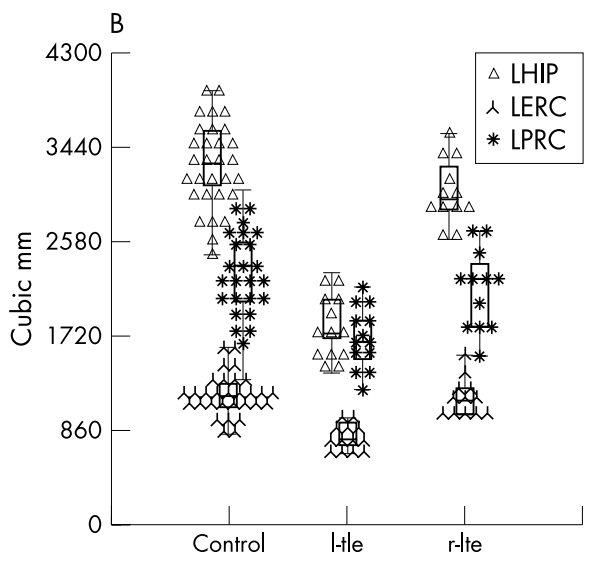

Figure 1 Box and whiskers plot showing the distribution of the volumes of the structures significantly atrophied in MTLE: the entorhinal cortex (ERC), the perirhinal cortex (PRC) and the hippocampus (Hip). (A) Distribution between groups (control, right MTLE (Rtle) and left MTLE (Ltle)) of the volumes of the ERC, PRC and Hip on the right side; and (B) distribution between groups of the left sided structures.
Left hippocampus

$\lambda=10.95, p<0.001$.

Tukey's: LTLE < Control = RTLE

Left perirhinal cortex

$\lambda=6.55, \mathrm{p}=0.01$.

Tukey's: LTLE $<$ Control $=$ RTLE

Left entorhinal cortex

$\lambda=24.76, p<0.001$.

Tukey's: LTLE < Control = RTLE 


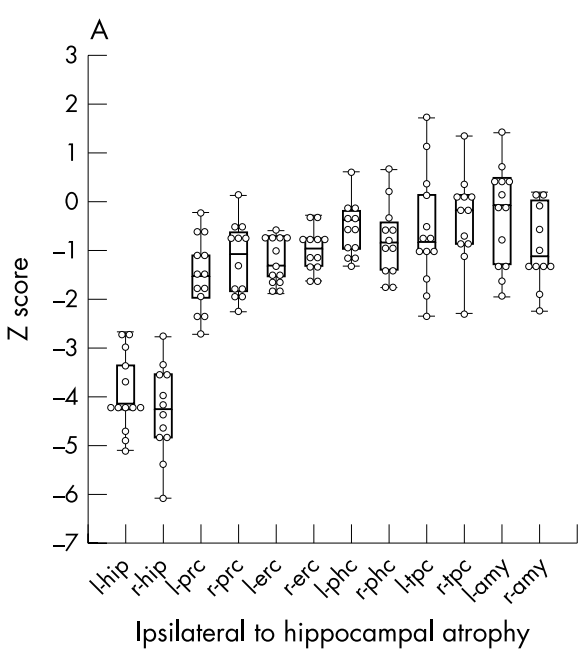

parahippocampal and temporopolar cortices, are less affected. For all medial temporal lobe structures, there was a significant difference in the asymmetry index between patients with right MTLE and controls, while patients with left TLE exhibited significant difference of the asymmetry indexes of the Hip, PRC, and ERC. This information can be used as additional information for lateralisation of seizures.

We did not observe significant correlations between the volumes of any of the structures analysed, nor did we observe significant difference between the degree of damage to the entorhinal and perirhinal cortex. These null results may reflect both the relatively small number of patients examined in this study and the variability found when studying an unselected set of consecutive patients rather than a more homogenous group matched for other factors. Nevertheless, as there is a large neural network involved in the generation and propagation of seizures in MTLE, ${ }^{20}$ it is also possible that MTLE reflects a heterogeneous population composed of different patterns of atrophy beyond the hippocampus according to the different sub-pattern of network most intensively activated.

In conclusion, our work extends the understanding of the extent of atrophy of the medial temporal lobe in patients with MTLE. Some issues related to the pattern of medial temporal lobe volumetric alterations require further studies. For instance, it is not currently possible to define the role of the cortical structures in the symptom presentation of medial MTLE, neither is it possible to predict which clinical factors may influence the presence and extent of medial temporal lobe damage. However, the observation and quantification of damage extending beyond the hippocampus may be helpful for understanding the lateralisation of seizures in patients with MTLE and may provide clues to further investigation of the role of temporal lobe cortical structures in the pathophysiology of medial temporal lobe epilepsy.

\section{Authors' affiliations}

L Bonilha, E Kobayashi, F Cendes, L M Li, Neuroimaging Laboratory, Department of Neurology, State University of Campinas, Brazil C Rorden, School of Psychology, University of Nottingham, UK Competing interest: none declared
Figure 2 Box and whiskers plot showing the distribution of the $Z$ scores of all structures analysed. (A) Z score of structures ipsilateral to the hippocampal atrophy; (B) Z score of structures contralateral to the hippocampal atrophy. R, right; L, left; PRC, perirhinal cortex; TPC, temporopolar cortex; ERC, entorhinal cortex; PHC,

parahippocampal cortex; Amy, amygdala; Hip, hippocampus.

\section{REFERENCES}

1 Johnston D, Amaral D. Hippocampus. In: Shepherd GM, ed. Synaptic organization of the brain. New York, Oxford: Oxford University Press, 1998:417-58.

2 Squire LR, Zola-Morgan S. The medial temporal lobe memory system. Science 1991;253:1380-6.

3 Falconer MA, Serafetinides EA, Corsellis JA. Etiology and pathogenesis of temporal lobe epilepsy. Arch Neurol 1964;10:233-248.

4 Meencke HJVG. Hippocampal sclerosis in epilepsy. In: Luders HO, ed. Epilepsy surgery. New York: Raven Press, 1991:705-15.

5 Bernasconi N, Bernasconi A, Caramanos Z, et al. Mesial temporal damage in temporal lobe epilepsy: a volumetric MRI study of the hippocampus, amygdala and parahippocampal region. Brain 2003;126:462-9.

6 Jutila L, Ylinen A, Partanen K, et al. MR volumetry of the entorhinal, perirhinal, and temporopolar cortices in drug-refractory temporal lobe epilepsy. AJNR Am J Neuroradiol 2001:22:1490-501.

7 Bernasconi N, Bernasconi A, Andermann F, et al. Entorhinal cortex in temporal lobe epilepsy: a quantitative MRI study. Neurology 1999;52:1870-6.

8 Bernasconi N, Bernasconi A, Caramanos Z, et al. Morphometric MRI analysis of the parahippocampal region in temporal lobe epilepsy. Ann NY Acad Sci 2000;911:495-500.

9 Bernasconi N, Bernasconi A, Caramanos Z, et al. Entorhinal cortex atrophy in epilepsy patients exhibiting normal hippocampal volumes. Neurology 2001:56:1335-9.

10 Salmenpera T, Kalviainen R, Partanen K, et al. Quantitative MRI volumetry of the entorhinal cortex in temporal lobe epilepsy. Seizure 2000;9:208-15.

11 Insausti R, Juottonen K, Soininen H, et al. MR volumetric analysis of the human entorhinal, perirhinal, and temporopolar cortices. AJNR Am J Neuroradiol 1998:19:659-71.

12 Pruessner JC, Li LM, Serles W, et al. Volumetry of hippocampus and amygdala with high-resolution MRI and three-dimensional analysis software: minimizing the discrepancies between laboratories. Cereb Cortex 2000; 10:433-42.

13 Commission on Classification and Terminology of the International League Against Epilepsy. Proposal for revised classification of epilepsies and epileptic syndromes. Epilepsia 1989;30:389-99.

14 Kobayashi E, Cendes F, Guerreiro CAM, et al. MRI abnormalities in familial temporal lobe epilepsy. Neurology 1999:52(Suppl 2):A545.

15 Engel J Jr. The timing of surgical intervention for mesial temporal lobe epilepsy: a plan for a randomized clinical trial. Arch Neurol 1999;56:1338-41.

16 Sled JG, Zijdenbos AP, Evans AC. A nonparametric method for automatic correction of intensity nonuniformity in MRI data. IEEE Trans Med Imaging 1998; 17:87-97.

17 Collins DL, Neelin P, Peters TM, et al. Automatic 3D intersubject registration of MR volumetric data in standardized Talairach space. J Comput Assist Tomogr 1994:18:192-205.

18 Salmenpera T, Kalviainen R, Partanen K, et al. MRI volumetry of the hippocampus, amygdala, entorhinal cortex, and perirhinal cortex after status epilepticus. Epilepsy Res 2000;40:155-70.

19 Cendes F, Andermann F, Gloor P, et al. MRI volumetric measurement of amygdala and hippocampus in temporal lobe epilepsy. Neurology 1993:43.719-25.

20 Spencer SS. Neural networks in human epilepsy: evidence of and implications for treatment. Epilepsia 2002;43:219-27. 